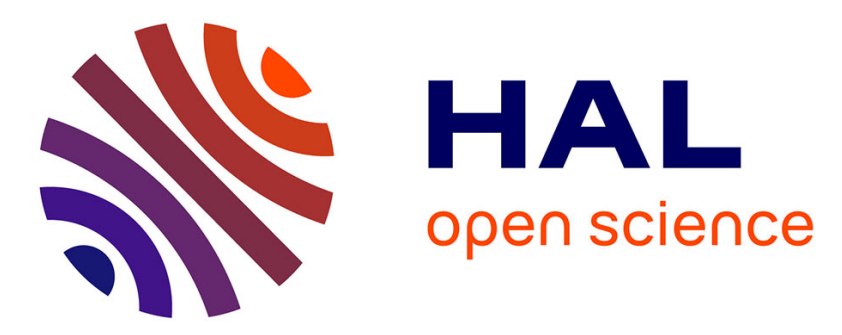

\title{
How long can excess pollution persist? The non-cooperative case
}

Pierre-Yves Hénin, Katheline Schubert

\section{To cite this version:}

Pierre-Yves Hénin, Katheline Schubert. How long can excess pollution persist? The non-cooperative case. Resource and Energy Economics, 2008, 30 (2), pp.277-293. 10.1016/j.reseneeco.2007.04.001. hal-00267762

\section{HAL Id: hal-00267762 \\ https://hal.science/hal-00267762}

Submitted on 28 Mar 2008

HAL is a multi-disciplinary open access archive for the deposit and dissemination of scientific research documents, whether they are published or not. The documents may come from teaching and research institutions in France or abroad, or from public or private research centers.
L'archive ouverte pluridisciplinaire HAL, est destinée au dépôt et à la diffusion de documents scientifiques de niveau recherche, publiés ou non, émanant des établissements d'enseignement et de recherche français ou étrangers, des laboratoires publics ou privés. 


\title{
How long can excess pollution persist? The non-cooperative case
}

\author{
Pierre-Yves HÉnIN \\ Katheline SCHUBERT ${ }^{* \dagger}$ \\ Université Paris 1 Panthéon-Sorbonne. Université Paris 1 Panthéon-Sorbonne.
}

March 2007

\begin{abstract}
This paper describes a world composed of two (groups of) countries, which derive their utility from a polluting activity and from the enjoyment of a common environmental quality. The initial situation is both suboptimal and unsustainable: pollution leads to a continuous deterioration of environmental quality. The two countries have heterogeneous preferences for the environment, which are private knowledge. This prevents the adoption of abatement policies negotiated between the two countries, because each one has a strong incentive to announce in every negotiation an arbitrarily low preference for the environment. The two countries then engage in a war of attrition, each of them postponing abatement policies, in the hope that the other will concede first and abate more. We study for how long the adjustment is postponed, according to initial conditions, the greenness of the greenest country, the possible range of preferences and the rates of discount and natural regeneration.
\end{abstract}

keywords: war of attrition, environmental negotiations, climate change

JEL classification: C72, Q20

${ }^{*}$ Corresponding author. Address: Centre d'Economie de la Sorbonne, 106-112 bd. de l'Hôpital, 75647 Paris Cedex 13, France. E-mail: schubert@univ-paris1.fr

${ }^{\dagger}$ We thank Martine Carré, Jean-François Jacques and Larry Karp for their helpful suggestions, criticisms and remarks. 


\section{Introduction}

International negotiations about environmental public goods are often disappointing, with advances closely followed by backward steps. In the field of climate change for example, recent events - particularly President Bush's rejection of the Kyoto Protocol- show that it is easier to postpone measures aimed at reducing greenhouse gas emissions than to reach an agreement on the sharing of efforts to reduce these emissions. We now have good reasons to expect that sizeable international pollution abatement will be further delayed, thus raising the question: how long can excess pollution persist?

The literature has tackled the question of international negotiations about environmental public goods in two ways.

The first approach analyses the problem in a cooperative framework and defines the mechanisms able to sustain cooperation (see for example Chander and Tulkens, 1992, 1995). But recent experiences in the field of climate change argue in favor of non-cooperative approaches. Moreover, we are interested in this paper in the mechanisms that allow non-cooperation to persist, thus preventing the emergence of stabilization.

The second approach consists in looking at the formation of environmental agreements from the point of view of non-cooperative game theory (see for example Barrett, 1994, 1998, Carraro and Siniscalco, 1993, Hoel, 1992). Most theoretical papers in this strand of the literature retain an assumption of homogeneous countries, and focus on the size of the coalitions that can emerge. Our intention is to study the behaviour of heterogeneous countries in a two countries set-up, thus eliminating the question of formation of coalitions.

We describe a world composed of two groups of countries, which derive their utility from a polluting activity but also from the enjoyment of a common environmental quality. The initial situation is commonly known as both suboptimal and unsustainable in the sense that global pollution is excessive and leads to a continuous deterioration of environmental quality over time. The main assumption is that the two groups of countries have different preferences as far as environmental quality is concerned. Apart from their divergence in the valuation of 
environmental assets, the two groups of countries are identical and emit the same amount of pollution in the business-as-usual pre-stabilization situation.

As far as climate change is concerned, these two groups of countries can be seen as the European countries on the one hand and the United States on the other hand. Their environmental consciousness is different, and so is their evaluation of the reality of the climate change problem. Moreover, they do not agree on the means that must be brought into play to tackle it. While European countries favor the Kyoto Protocol, the United States is skeptical and seems to rely on technical progress to deal with this problem, without the need of additional initiatives that could jeopardize U.S. growth.

The preference for the environment cannot be reduced to a subjective environmental consciousness. It also reflects the marginal damage caused by the worsening of environmental quality or, equivalently, the marginal benefit of an improvement in environmental quality, and countries differ in this respect. It is difficult for either group of countries ${ }^{1}$ to know the level of preference for the environment of the other, since this preference is a mix of subjective environmental consciousness and of objective marginal damage. In addition, each country behaves strategically and is tempted to announce biased preferences in negotiations in order to manipulate information.

The environmental preference is therefore private knowledge. This asymmetry of information prevents the emergence of a negotiated solution, because each country has a strong incentive to announce an arbitrarily low preference for the environment. The two countries then engage in a war of attrition, each of them postponing the implementation of measures intended to reduce pollution with the hope that the other one will concede first.

War of attrition models have been applied to various issues, such as labor strikes, biological competition, industrial organization and, in the political economy field, fiscal stabilization. Bac (1996) underlines their relevance in the case of environmental questions. In this paper we adopt a framework closely related to war of attrition models applied to fiscal stabilization (Alesina and Drazen, 1991, Casella and Eichengreen, 1996, Carré, 2000), because our case has in common

\footnotetext{
${ }^{1}$ In the following, we will content ourselves with speaking of two countries instead of two groups of countries, for ease of reference.
} 
with these models its stock-flow structure, the stock being a public good and the flow a private advantage. Compared to these models, the game we consider in this paper nevertheless has several distinctive features.

First, in the fiscal stabilization problem, stabilization can always be postponed; it is not the case in our model where if nothing is done in finite time, the environmental capital reaches a minimal threshold that cannot be passed. Carré (2000), building on Alesina and Drazen's model, introduces an exogenous deadline such that if fiscal stabilization has not occurred before this date a penalty is paid by all players. Our model cannot be reduced to this framework, because it is impossible here for the agents to decide to pass beyond the deadline.

Secondly, due to the lack of a supra-national authority, the burden of stabilization cannot be shared, even unequally, between the two players $^{2}$. We consider that one of the countries, the loser, can decide unilaterally to try to stabilize environmental quality. We also show that if nothing is done to stabilize environmental quality before a certain limit date, stabilization requires that both countries reduce pollution. There are then two games: a first one where environmental conditions are such that one country can stabilize alone, and a second one where environmental conditions are so deteriorated that both countries must reduce pollution in order to achieve stabilization.

The paper is organized as follows. Section 2 sets the framework and the basic assumptions of the model. In Section 3, information is assumed to be asymmetric. Considering the case where an immediate stabilization would occur under perfect information, we study how long the adjustment will be postponed, and the influence of the parameters that characterize each country. We show that there exists a critical parameter of preference for the environment, which depends on initial conditions, on the possible range of preferences and on the rates of discount and natural regeneration. If the parameter of preference of the greenest country is greater than this critical value, the optimal stabilization date is, loosely speaking, not too far, and this country will stabilize environmental quality alone. If the environmental parameter is less than

\footnotetext{
${ }^{2}$ The absence of any supra-national authority provides a strong rationale for representing the global environmental problem as a war of attrition. This rationale is not as strong in the case of fiscal stabilization issues.
} 
the critical value, stabilization will be late and both countries will have to reduce pollution. In that case, if the discount rate is high enough, an accumulation of concessions at the limit date by which the natural capital reaches its minimal threshold occurs. If the discount rate is even higher, a jump in the concession function can appear. Section 4 concludes and discusses possible extensions

\section{The model}

\subsection{The general framework}

Two countries derive their utility from consumption and from the enjoyment of a common environmental quality. Emissions of pollution of country $i, p_{i}(t)$, are strictly proportional to economic activity and hence to consumption. From an initial level $q_{0}$, global environmental quality $q(t)$ is deteriorated by the polluting activity of both countries but regenerates at a constant rate $\delta>0$. Its law of motion is then

$$
\dot{q}(t)=\delta q(t)-\left(p_{1}(t)+p_{2}(t)\right) .
$$

Environmental quality is defined as the difference between the current level of natural capital and the minimal level necessary to support human life, below which natural capital cannot fall. The constraint $q(t) \geq 0$ must then be satisfied.

The instantaneous utility of country $i, u_{i}(t)$, is supposed linear in its two arguments, country i's pollution and global environmental quality ${ }^{3}$ :

$$
u_{i}(t)=p_{i}(t)+\theta_{i} q(t) \quad i=1,2,
$$

where $\theta_{i}$ is the "preference for the environment" of country $i$ and characterizes the "greenness" of preferences. The intertemporal welfare of country $i$ is the sum of the instantaneous utilities discounted at a constant rate $\gamma>0$, supposed identical for the two countries.

\footnotetext{
${ }^{3}$ This linearity assumption is usual in the war of attrition literature because it enables a complete analytical characterization of the solution. But it has obvious drawbacks, mainly the fact that utility remains finite as environmental quality approaches zero, and so nothing prevents the optimal choice of a zero environmental quality. But history reminds us that such a choice can actually exist, through, for example, the famous case of Easter Island.
} 
The business-as-usual pollution level is assumed to be constant and identical for the two countries $^{4}$, and is denoted by $p>0$. Total pollution is then $2 p$ in BAU. Thus, the preference parameter is the only feature that distinguishes one country from the other in the BAU situation. In BAU, the environmental quality level at date $t$ is given by:

$$
q_{\mathrm{BAU}}(t)=q_{0} e^{\delta t}+\frac{2 p}{\delta}\left(1-e^{\delta t}\right)
$$

We focus on the case of excessive initial pollution: we suppose $p>\frac{\delta}{2} q_{0}$, so that environmental quality deteriorates if nothing is done to reduce pollution. Thus the world economy follows, in the BAU situation, an unsustainable pollution path.

In order to stabilize environmental quality at time $t$, global pollution must be reduced to the level $P(t)$ such that $\dot{q}(t)=0$, which requires that it becomes just equal to natural regeneration:

$$
P(t)=\delta q_{\mathrm{BAU}}(t)
$$

$P(t)$ is the sustainable pollution level. Obviously, the global effort required to stabilize environmental quality, equal to $2 p-P(t)$ at time $t$, is all the larger since stabilization occurs at a later date.

Within the non-cooperative framework considered in this paper, a solution to the environmental problem consists in finding i) the country that makes the first effort of stabilization, and ii) the date at which this occurs 5 .

\section{$2.2 \quad$ The limit dates}

We define a first limit date $T_{m}$ as the last date at which one country is able to stabilize environmental quality by itself. At $T_{m}$, the required reduction of total pollution $\left(2 p-P\left(T_{m}\right)\right)$ equals the

\footnotetext{
${ }^{4}$ The assumption of exogenous, constant and identical BAU emissions for the two countries is admittedly unrealistic, but we have made it in order to avoid the mix of two different problems: (1) the determination of the optimal path of pollution of each country before they become aware of the necessity to stabilize environmental quality (the "historical" or BAU behavior of each country); and (2) the behavior of each country after it realizes the necessity to stabilize environmental quality: will it stabilize and, if yes, when? In other words, this assumption is necessary to reduce the problem to one dimension: the choice of one date.

${ }^{5}$ Notice that our purpose is different from that of Dockner and Van Long (1993), who study, in the linearquadratic case, an optimal control of pollution problem with perfect information. We do not try to answer the question of the optimal reduction of pollution of each country at each date, in order to concentrate on the war of attrition problem.
} 
total pollution by one country $(p)$. Thus the country that reduces emissions first (the loser, to follow the terminology usual in war of attrition models) at time $T_{m}$ must reduce its pollution to zero in order to stabilize environmental quality, while the other country (the winner) continues to pollute $p$. Date $T_{m}$ is then defined by

$$
P\left(T_{m}\right)=p=\delta q_{\mathrm{BAU}}\left(T_{m}\right) \Leftrightarrow q_{\mathrm{BAU}}\left(T_{m}\right)=\frac{p}{\delta} \Leftrightarrow q_{0} e^{\delta T_{m}}+\frac{2 p}{\delta}\left(1-e^{\delta T_{m}}\right)=\frac{p}{\delta},
$$

from which we obtain

$$
T_{m}=\frac{1}{\delta} \ln \frac{p}{2 p-\delta q_{0}}
$$

This date is strictly positive if and only if $p<\delta q_{0}$. In the opposite case, a single country cannot stabilize. So we suppose this condition fulfilled in the rest of the paper. We then have, in the initial situation:

Assumption $1 \frac{\delta}{2} q_{0}<p<\delta q_{0}$. The initial environmental quality $q_{0}$ can sustain one unrestricted polluting country, but not two, i.e. global pollution emissions greater than $p$ but less than $2 p$.

The higher the initial pollution $p$, the closer the limit date $T_{m}$, and the higher the initial environmental quality $q_{0}$, the later $T_{m}$.

If none of the two countries decides to stabilize before date $T_{m}$, it becomes too late for one country to stabilize by itself. After $T_{m}$, if the greenest country decides to concede at a date $\widetilde{T}$, it must cut its pollution to zero, but that will not be enough, and stabilization of environmental quality will only take place if the other country also reduces its pollution, to a level just equal to the natural regeneration capacity. The problem is then to find when the winner will accept to bear its part of the burden of adjustment, actually smaller than the part of the loser: as soon as the loser concedes (that is to say at $\widetilde{T}$ ), later, or never?

Let us suppose that the loser decides to reduce its pollution to zero at $\widetilde{T}>T_{m}$. From $\widetilde{T}$ to the date $\tau \geq \widetilde{T}$ at which the winner decides to bear its part of the burden, environmental quality evolves according to the following equation:

$$
\dot{q}(t)=\delta q(t)-p, \quad \widetilde{T} \leq t \leq \tau,
$$


and so environmental quality is given by

$$
q(t)=q_{\mathrm{BAU}}(\widetilde{T}) e^{\delta(t-\widetilde{T})}+\frac{p}{\delta}\left(1-e^{\delta(t-\widetilde{T})}\right), \quad \widetilde{T} \leq t \leq \tau .
$$

We get, by replacing $q_{\mathrm{BAU}}(\widetilde{T})$ by its value given by equation (3),

$$
q(t)=q_{0} e^{\delta t}+\frac{p}{\delta}\left(1+e^{\delta(t-\widetilde{T})}-2 e^{\delta t}\right), \quad \widetilde{T} \leq t \leq \tau .
$$

If the winner goes on polluting $p$ after $\widetilde{T}$, environmental quality will be exhausted at a date $\widetilde{t}$ defined by $q(\widetilde{t})=0$, and we easily see that $\widetilde{t}$ depends on $\widetilde{T}$ and is given by

$$
e^{-\delta \widetilde{t}}=e^{-\delta T_{m}}-e^{-\delta \widetilde{T}}
$$

This date is an endogenous deadline, at which natural capital is at its minimal level ${ }^{6}$, despite a unilateral pollution abatement of the greenest country at $\widetilde{T}$.

Nevertheless, there exists another limit date $T_{x}>T_{m}$ at which environmental quality is exhausted while both countries have done nothing to reduce pollution:

$$
q_{\mathrm{BAU}}\left(T_{x}\right)=0 \Leftrightarrow q_{0} e^{\delta T_{x}}+\frac{2 p}{\delta}\left(1-e^{\delta T_{x}}\right) \Leftrightarrow e^{\delta T_{x}}=\frac{2 p}{2 p-\delta q_{0}}=2 e^{\delta T_{m}}
$$

from which we deduce

$$
T_{x}=T_{m}+\frac{\ln 2}{\delta}
$$

This date is an exogenous deadline, before which something must be done to stabilize environmental quality if the countries want to avoid its exhaustion.

\subsection{The perfect information case}

When information is perfect, the parameters of preference for the environment of the two countries $\theta_{i}$ and $\theta_{j}$ are common knowledge. The greenest country (higher $\theta$ ) then knows, in a non-cooperative framework, that it will bear the whole (if it is sufficient) or the greatest part (if it is not) of the cost of stabilization. The only variable that must be determined in this

\footnotetext{
${ }^{6}$ Notice that because of our choice of a linear utility function we do not impose an infinite penalty on welfare in the case of an environmental quality equal to zero. The incentive to stabilize before the deadline would have of course been greater in the case of a utility function satisfying the Inada condition.
} 
case is the date of stabilization. We study in Appendix $\mathrm{A}^{7}$ the perfect information case as a benchmark for the case with asymmetric information. Let $\gamma$ be the rate of time preference of the two countries. We show that if the parameter of preference for the environment of the greenest country is less than impatience corrected for natural regeneration $(\gamma-\delta)$, stabilization will never be performed and the two countries will exhaust environmental quality. Conversely, if this parameter is higher than impatience corrected for natural regeneration, stabilization will occur at once.

With asymmetric information, stabilization will take place at best at the same time as when information is perfect, never earlier. Then we restrict ourselves to the case where, when information is perfect, the countries do not choose to let the natural capital reach its minimal level, and we reduce for this reason the range of possible preferences such that the smallest possible preference parameter, denoted $\underline{\theta}$, is greater than impatience corrected for natural regeneration:

Assumption $2 \underline{\theta}>\gamma-\delta$.

\section{The consequences of asymmetric information}

We now consider the case where information is asymmetric in the sense that the value of the preference for the environment of each country is private information. We suppose that from the point of view of country $i$, the preference for the environment of country $j, \theta_{j}$, is drawn from a distribution $F\left(\theta_{j}\right)$, with lower and upper bounds $\underline{\theta}$ and $\bar{\theta}$. The distribution $F(\theta)$ is known and a priori supposed common to the two countries. $f(\theta)$ is the associated density function. As far as $\theta_{j}$ is unknown for $i$, we must characterize non-cooperative strategies of unilateral reduction of emissions by concession by one of the two countries, as a function of its conjecture about the other country's behaviour.

As usual in war of attrition models (Alesina and Drazen, 1991), country $i$ will decide to concede at time $T$ rather than at time $T+d T$ if the expected gain associated with the fact that

\footnotetext{
${ }^{7}$ The Appendix to this paper can be provided by the authors on request, or can be downloaded as a pdf document from http://eurequa.univ-paris1.fr/membres/schubert/publications.htm.
} 
the other country might decide to concede during $d T$ is smaller than the advantage it has in conceding immediately.

The problem is different when the optimal date of concession $T$ is smaller or higher than the limit date $T_{m}$. In the first case one country is able to stabilize alone, while in the second case the other one must make an effort as well. We then study these two cases separately.

\subsection{Concession before $T_{m}$}

\subsubsection{The optimal date of concession}

The country that concedes at time $T \leq T_{m}$ (the loser) reduces its pollution to the level $\delta q_{\mathrm{BAU}}(T)-$ $p$ in order to achieve stabilization. Its utility is $u_{i}^{L 1}(t)=\left(\delta+\theta_{i}\right) q_{\mathrm{BAU}}(T)-p \forall t \geq T$, and its intertemporal welfare from $T \leq T_{m}$ is

$$
V_{i}^{L 1}(T)=\int_{T}^{\infty} e^{-\gamma(t-T)} u_{i}^{L 1}(t) d t=\frac{u_{i}^{L 1}(T)}{\gamma}=\frac{\left(\delta+\theta_{i}\right) q_{\mathrm{BAU}}(T)-p}{\gamma} .
$$

The country that has not conceded when the other one concedes at time $T$ (the winner) has a utility $u_{i}^{W 1}(t)=p+\theta_{i} q_{\mathrm{BAU}}(T) \forall t \geq T$, and its intertemporal welfare from $T$ is

$$
V_{i}^{W 1}(T)=\int_{T}^{\infty} e^{-\gamma(t-T)} u_{i}^{W 1}(t) d t=\frac{u_{i}^{W 1}(T)}{\gamma}=\frac{p+\theta_{i} q_{\mathrm{BAU}}(T)}{\gamma}
$$

The current cost of a unilateral concession at time $T \leq T_{m}$ is then $u_{i}^{W 1}(T)-u_{i}^{L 1}(T)=$ $2 p-\delta q_{\mathrm{BAU}}(T)$, while the intertemporal cost is $V_{i}^{W 1}(T)-V_{i}^{L 1}(T)=\frac{2 p-\delta q_{\mathrm{BAU}}(T)}{\gamma}$.

Country $i$ must choose its strategy of concession, as a function of its expectation of the probabilities of concession of the other country. Let $h_{j}(t)$ denote the density of the random date of concession of country $j$ and $H_{j}(t)$ its distribution function. The intertemporal expected utility of country $i$ is then

$$
\begin{aligned}
E U_{i}^{1}(T)= & {\left[1-H_{j}(T)\right]\left[\int_{0}^{T} e^{-\gamma t} u_{i}(t) d t+e^{-\gamma T} V_{i}^{L 1}(T)\right] } \\
& +\int_{0}^{T} h_{j}(t)\left[\int_{0}^{t} e^{-\gamma s} u_{i}(s) d s+e^{-\gamma t} V_{i}^{W 1}(t)\right] d t .
\end{aligned}
$$

Country $i$ seeks to maximize its intertemporal expected utility, subject to the constraint $q_{\mathrm{BAU}}(T) \leq q_{\mathrm{BAU}}\left(T_{m}\right)$ 
If this problem admits an interior solution the optimal conceding time satisfies the first order condition $\frac{d E U_{i}^{1}(T)}{d T}=0$, i.e.

$$
\begin{aligned}
& -h_{j}(T)\left[\int_{0}^{T} e^{-\gamma t} u_{i}(t) d t+e^{-\gamma T} V_{i}^{L 1}(T)\right] \\
& +\left[1-H_{j}(T)\right]\left[e^{-\gamma T} u_{i}(T)-\gamma e^{-\gamma T} V_{i}^{L 1}(T)+e^{-\gamma T} \frac{d V_{i}^{L 1}(T)}{d T}\right] \\
& +h_{j}(T)\left[\int_{0}^{T} e^{-\gamma s} u_{i}(s) d s+e^{-\gamma T} V_{i}^{W 1}(T)\right]=0,
\end{aligned}
$$

which states that the marginal benefit of conceding at time $T$ rather than at time $T+d T$ is equal to the marginal benefit of waiting during $d T$. Following the presentation of Casella and Eichengreen (1996), the optimality condition can be written as

$$
-u_{i}(T)+\gamma V_{i}^{L 1}(T)-\frac{d V_{i}^{L 1}(T)}{d T}=\frac{h_{j}(T)}{1-H_{j}(T)}\left[V_{i}^{W 1}(T)-V_{i}^{L 1}(T)\right],
$$

or, using the definitions of the different terms of welfare:

$$
\left(2 p-\delta q_{\mathrm{BAU}}(T)\right) \frac{\theta_{i}+\delta-\gamma}{\gamma}=\frac{h_{j}(T)}{1-H_{j}(T)} \frac{2 p-\delta q_{\mathrm{BAU}}(T)}{\gamma} .
$$

The left-hand side of equations (12) and (13) is the marginal benefit of conceding at time $T$ rather than at time $T+d T$, for country $i$, while the right-hand side is the marginal benefit of waiting during $d T$, equal to the probability (conjectured by $i$ ) that $j$ concedes between $T$ and $T+d T$ knowing that it has not conceded before, multiplied by the cost of a unilateral concession at time $T$. We denote the marginal benefit of conceding $B_{i}^{1}(T)$ :

$$
B_{i}^{1}(T)=\left(2 p-\delta q_{\mathrm{BAU}}(T)\right) \frac{\theta_{i}+\delta-\gamma}{\gamma} .
$$

It is strictly positive by assumption 2, and besides it is obviously increasing in $T$, as BAU environmental quality is decreasing in $T$.

One easily checks that the second order condition is satisfied, which ensures that the solution is a maximum:

$$
\frac{d^{2} E U_{i}(T)}{d T d \theta_{i}}=-\frac{1}{\gamma} e^{-\gamma T}\left(2 p-\delta q_{\mathrm{BAU}}(T)\right)\left[1-H_{j}(T)\right]<0 .
$$

Equation (13) reduces to

$$
\frac{h_{j}(T)}{1-H_{j}(T)}=\theta_{i}+\delta-\gamma
$$


which defines the optimal time of concession for country $i$.

If the problem of country $i$ does not admit an interior solution, the optimal time of concession is $T_{m}$ and $\frac{d E U_{i}(T)}{d T}>0 \forall T<T_{m}$, which means that country $i$ is better-off by delaying stabilization for as long as possible. Then, country $i$ can either adopt the corner solution $T_{m}$ or pass to the second game, where it cannot stabilize by itself. This possibility will be studied after we complete the characterization of the interior solution.

\subsubsection{The symmetric Nash equilibrium}

We consider here the symmetric Nash equilibrium in which the two countries concede following the same function $T(\theta)^{8}$.

Remark 1 If a country has the highest possible parameter of preference $\bar{\theta}$, it concedes immediately, provided that its marginal benefit from conceding is positive, or as soon as its marginal benefit becomes positive if this is not the case immediately. This property results from the fact that such a country knows with certainty that any other country will wait. It provides us with a boundary condition for the determination of the concession function: $T(\bar{\theta})=0$.

Proposition 1 If the parameters of preference for the environment are uniformly distributed on $[\underline{\theta}, \bar{\theta}]$, the symmetric Nash equilibrium of the first game (the game in which one country can stabilize environmental quality alone) has the following characteristics:

(i) when an interior solution exists and under the boundary condition $T(\bar{\theta})=0$, the optimal conceding time for a parameter of preference $\theta$ is

$$
T(\theta)=\frac{1}{\underline{\theta}+\delta-\gamma}\left[\ln \frac{\theta+\delta-\gamma}{\bar{\theta}+\delta-\gamma}-\ln \frac{\theta-\underline{\theta}}{\bar{\theta}-\underline{\theta}}\right]
$$

(ii) an interior solution exists if and only if $\theta \in\left[\theta_{m}, \bar{\theta}\right]$, where $\theta_{m}$ is the limit parameter of preference corresponding to the limit date of concession $T_{m}$, and is given by

$$
\theta_{m}=\frac{\underline{\theta}(\bar{\theta}+\delta-\gamma) e^{(\underline{\theta}+\delta-\gamma) T_{m}}-(\gamma-\delta)(\bar{\theta}-\underline{\theta})}{(\bar{\theta}+\delta-\gamma) e^{(\underline{\theta}+\delta-\gamma) T_{m}}-(\bar{\theta}-\underline{\theta})} .
$$

\footnotetext{
${ }^{8}$ Within this model, there may be asymmetric equilibria in which countries behave according to different functions $T(\theta)$, even with the assumption of symmetry of the conjectured distribution of the preference parameters. In particular, there may be asymmetric equilibria in which one country concedes immediately. We do not investigate such equilibria since we are interested in showing that delaying stabilization can be optimal.
} 
Then $T(\theta)$ decreases from $T\left(\theta_{m}\right)=T_{m}$ to $T(\bar{\theta})=0$, and is convex $\left(T^{\prime \prime}(\theta)>0 \forall \theta \in\left[\theta_{m}, \bar{\theta}\right]\right)$;

(iii) for $\theta \in\left[\underline{\theta}, \theta_{m}\left[\right.\right.$, the solution is the corner solution $T(\theta)=T_{m}$.

Proof. Equation (15) defines the optimal date of concession for country $i$, as a function of its own preference for the environment $\theta_{i}$ and of its expectation of the distribution of the date of concession of the other country $H_{j}(T)$. But the distribution $H_{j}(T)$ is not known, and equation (15) cannot be used directly. Bliss and Nalebuff (1984) show that there exists a relation between the unknown $H_{j}(T)$ and the known $F(\theta)$, the distribution of the parameters of preference $\theta$, because the optimal date of concession is monotonic in $\theta$. Precisely, the probability that country $j$ will not concede before time $T, 1-H_{j}(T)$, is also the probability that its parameter of preference for the environment $\theta_{j}$ is smaller than the parameter $\theta$ associated with time $T$, that is $F(\theta)$. So we have

$$
1-H_{j}(T)=1-H_{j}(T(\theta))=F(\theta) .
$$

The densities are also linked: we obtain, by derivation of the preceding equation,

$$
-h_{j}(T) T^{\prime}(\theta)=f(\theta)
$$

One can deduce the probability (conjectured by $i$ ) that $j$ concedes between $T$ and $T+d T$ knowing that it has not conceded before:

$$
\frac{h_{j}(T)}{1-H_{j}(T)}=-\frac{f(\theta)}{F(\theta)} \frac{1}{T^{\prime}(\theta)}
$$

which yields, using equation (15),

$$
T^{\prime}(\theta)=-\frac{f(\theta)}{F(\theta)} \frac{1}{\theta+\delta-\gamma}<0
$$

In the case of a uniform distribution of the $\theta$ we have $f(\theta)=\frac{1}{\bar{\theta}-\underline{\theta}}$ and $F(\theta)=\frac{\theta-\underline{\theta}}{\bar{\theta}-\underline{\theta}}$. So

$$
T^{\prime}(\theta)=-\frac{1}{(\theta-\underline{\theta})(\theta+\delta-\gamma)},
$$

which integrates into

$$
T(\theta)=\frac{1}{\underline{\theta}+\delta-\gamma}\left[\ln \frac{\theta+\delta-\gamma}{a}-\ln \frac{\theta-\underline{\theta}}{b}\right] .
$$


Then, Remark 1 allows us to use the boundary condition $T(\bar{\theta})=0$, and so to fully characterize the optimal conceding time for a parameter of preference $\theta$ (equation (16)). This proves (i).

We easily see that $\lim _{\theta \backslash \underline{\theta}} T(\theta) \rightarrow+\infty$. But the solution given by equation (16) is valid only for parameters of preference $\theta$ such that $T(\theta)<T_{m}$. We then calculate the limit parameter of preference $\theta_{m}$ corresponding to the limit date of concession $T_{m}$ (more precisely, $T_{m}-\varepsilon$ with $\varepsilon$ arbitrarily small). We have

$$
\begin{aligned}
T\left(\theta_{m}\right)=T_{m} & \Leftrightarrow \frac{1}{\underline{\theta}+\delta-\gamma}\left[\ln \frac{\theta_{m}+\delta-\gamma}{\bar{\theta}+\delta-\gamma}-\ln \frac{\theta_{m}-\underline{\theta}}{\bar{\theta}-\underline{\theta}}\right]=\frac{1}{\delta} \ln \frac{p}{2 p-\delta q_{0}} \\
& \Leftrightarrow \frac{\theta_{m}+\delta-\gamma}{\theta_{m}-\underline{\theta}}=\frac{\bar{\theta}+\delta-\gamma}{\bar{\theta}-\underline{\theta}}\left(\frac{p}{2 p-\delta q_{0}}\right)^{\frac{\hat{\theta+\delta-\gamma}}{\delta}} \\
& \Leftrightarrow \frac{\theta_{m}+\delta-\gamma}{\theta_{m}-\underline{\theta}}=\frac{\bar{\theta}+\delta-\gamma}{\bar{\theta}-\underline{\theta}} e^{(\underline{\theta}+\delta-\gamma) T_{m}},
\end{aligned}
$$

from which we easily deduce (17).

We have $T^{\prime \prime}(\theta)=-\left(\frac{1}{\theta-\underline{\theta}}+\frac{1}{\theta+\delta-\gamma}\right) T^{\prime}(\theta)>0$. This completes the proof of (ii).

Finally, the proof of (iii) is straightforward.

$\theta_{m}$, given by equation (17), depends on the parameters of the model (range of preferences, social discount rate, natural regeneration rate) but also on the initial situation of the world economy in terms of pollution and environmental quality, through $T_{m}$.

Using equations (5) and (17), one can easily show that

$$
\left\{\begin{array} { l } 
{ \frac { \partial T _ { m } } { \partial p } < 0 } \\
{ \frac { \partial \theta _ { m } } { \partial p } > 0 }
\end{array} \text { and } \quad \left\{\begin{array}{l}
\frac{\partial T_{m}}{\partial q_{0}}>0 \\
\frac{\partial \theta_{m}}{\partial q_{0}}<0 .
\end{array}\right.\right.
$$

The higher the business-as-usual pollution and the smaller the initial environmental quality, the nearer the limit date $T_{m}$ and the higher the limit parameter of preference for the environment $\theta_{m}$. In other words, if the business-as-usual level of pollution is high or the initial environmental quality low, $T_{m}$ will be close to now and $\theta_{m}$ close to $\bar{\theta}$. A stabilization by a single country will occur only if the greenest country is very green, since it requires a preference parameter close to $\bar{\theta}$. If that is the case, it will occur quickly, since the date beyond which stabilization by a single country is impossible is close to now. 
We have

$$
\frac{\partial \theta_{m}}{\partial \bar{\theta}}=\frac{(\underline{\theta}+\delta-\gamma)^{2}}{\bar{\theta}+\delta-\gamma}>0
$$

This means that when the range of preferences is larger on the right everything being equal, the parameter of preference beyond which stabilization occurs before $T_{m}$ is higher. If the two countries believe that the other one could be very green, stabilization is less likely to occur before $T_{m}$.

One can also show that $\frac{\partial \theta_{m}}{\partial(\bar{\theta}-\underline{\theta})}>0$. So a larger range of the possible values of the parameters of preference for the environment (a larger dispersion of preferences) leads to an increase of the parameter of preference beyond which stabilization occurs before $T_{m}$.

Finally, it is possible to show that $\theta_{m}$ increases with the discount rate, everything being equal (the proof is relegated to Appendix $\mathrm{B}^{9}$ ). This means that when the two countries are very impatient, a stabilization by a single country will occur only if the greenest country is very green; if this is not the case, it is rational to delay stabilization at least to the point where one country can no longer stabilize alone.

\subsection{Concession between $T_{m}$ and $T_{x}$}

Suppose now that an interior solution to the first game does not exist. Is it then optimal for country $i$ to adopt the corner solution of the first game and stabilize at $T_{m}$, or is it optimal to postpone stabilization again and play the second game? Its choice will depend only on the value of the second game, compared to the corner value of the first one. We first study the solution of the second game and then answer this question.

\subsubsection{The optimal date of concession in the second game}

If none of the two countries has conceded at time $T_{m}$, they reveal that their parameter of preference is smaller than $\theta_{m}$. The range of the parameters of preference is thus reduced to $\left[\underline{\theta}, \theta_{m}[\right.$. Now, a single country cannot stabilize alone.

\footnotetext{
${ }^{9}$ See footnote 7 .
} 
Let us suppose that the loser cuts its pollution to zero at $T>T_{m}$. It reveals then that its parameter of preference for the environment is higher than that of the other country. The winner no longer faces any uncertainty and is in a situation of perfect information. Appendix $\mathrm{A}^{10}$ studies the problem the winner faces and shows how its intertemporal welfare evolves with the date at which it chooses to stabilize. Here, by assumption 2, intertemporal welfare is a strictly decreasing function of this date, and the winner chooses to stabilize immediately, as soon as the loser has cut its pollution to zero ${ }^{11}$. The problem is then to find when the loser will decide to do it.

The instantaneous utilities and intertemporal welfares of the two countries from $T>T_{m}$ are $u_{i}^{L 2}(T), V_{i}^{L 2}(T), u_{i}^{W 2}(T)=\left(\delta+\theta_{i}\right) q_{\mathrm{BAU}}(T)$ and $V_{i}^{W 2}(T)=\frac{\left(\delta+\theta_{i}\right) q_{\mathrm{BAU}}(T)}{\gamma}$, and the intertemporal cost of conceding is

$$
V_{i}^{W 2}(T)-V_{i}^{L 2}(T)=\frac{\delta q_{\mathrm{BAU}}(T)}{\gamma}, \quad T>T_{m}
$$

The expected intertemporal utility of country $i$ is

$$
\begin{aligned}
E U_{i}^{2}(T)= & {\left[1-H_{j}(T)\right]\left[\int_{0}^{T} e^{-\gamma t} u_{i}(t) d t+e^{-\gamma T} V_{i}^{L 2}(T)\right] } \\
& +\int_{0}^{T} h_{j}(t)\left[\int_{0}^{t} e^{-\gamma s} u_{i}(s) d s+e^{-\gamma t} V_{i}^{W 2}(t)\right] d t
\end{aligned}
$$

and country $i$ seeks to maximize $E U_{i}^{2}(T)$ subject to the constraint $q_{\mathrm{BAU}}(T) \geq 0$.

If we denote by $\mu$ the Lagrange multiplier associated with the constraint, the first order condition of this problem is

$$
\frac{d E U_{i}^{2}(T)}{d T}+\mu(T) \dot{q}(T)=0
$$

together with the complementarity slackness condition:

$$
\mu(T) \geq 0, q_{\mathrm{BAU}}(T) \geq 0, \text { and } \mu(T) q_{\mathrm{BAU}}(T)=0 .
$$

Let us first study the corner solution, characterized by $\mu(T)>0, q_{\mathrm{BAU}}(T)=0$ and $\frac{d E U_{i}^{2}(T)}{d T}+\mu(T) \dot{q}(T)=0$.

\footnotetext{
${ }^{10}$ See footnote 7 .

${ }^{11}$ Thus avoiding the moral hazard problem arising when the loser's concession only allows the winner to pollute later.
} 
We have

$\frac{d E U_{i}^{2}(T)}{d T}=h_{j}(T) e^{-\gamma T}\left[V_{i}^{W 2}(T)-V_{i}^{L 2}(T)\right]+\left[1-H_{j}(T)\right] e^{-\gamma T}\left[u_{i}(T)-\gamma V_{i}^{L 2}(T)+\frac{d V_{i}^{L 2}(T)}{d T}\right]$

or, when we replace the different functions by their expressions:

$$
\frac{d E U_{i}^{2}(T)}{d T}=h_{j}(T) e^{-\gamma T}\left[\frac{\delta q_{\mathrm{BAU}}(T)}{\gamma}\right]+\left[1-H_{j}(T)\right] e^{-\gamma T}\left[p-\left(2 p-\delta q_{\mathrm{BAU}}(T)\right) \frac{\theta_{i}}{\gamma}\right] .
$$

The conditions characterizing the corner solution are then

$$
\left\{\begin{array}{l}
q_{\mathrm{BAU}}(T)=0 \\
\mu(T)=\left[1-H_{j}(T)\right] \frac{e^{-\gamma T}}{\gamma}\left[\frac{\gamma}{2}-\theta_{i}\right]>0,
\end{array}\right.
$$

and the second of these equations gives us the values of the parameters of preference for which the corner solution prevails: $\theta_{i}<\frac{\gamma}{2}$.

For $\theta_{i} \geq \frac{\gamma}{2}$, the relevant solution is the interior one. The first order condition $\frac{d E U_{i}^{2}(T)}{d T}=0$ can then be written as

$$
\left(2 p-\delta q_{\mathrm{BAU}}(T)\right) \frac{\theta_{i}}{\gamma}-p=\frac{h_{j}(T)}{1-H_{j}(T)} \frac{\delta q_{\mathrm{BAU}}(T)}{\gamma} .
$$

The second order condition is satisfied.

As in the first game, the left-hand side of equation (26) is the marginal benefit of conceding during $d T$ while the right-hand side is the marginal benefit of waiting. We denote the marginal benefit of conceding for country $i B_{i}^{2}(T)$. We easily see that

$$
B_{i}^{2}(T)=\left(2 p-\delta q_{\mathrm{BAU}}(T)\right) \frac{\theta_{i}}{\gamma}-p=\left(2 p-\delta q_{0}\right)\left(\frac{\theta_{i}}{\gamma} e^{\delta T}-e^{\delta T_{m}}\right) .
$$

$B_{i}^{2}(T)$ is obviously increasing in $T$. Besides, it has the sign of the second term in brackets.

We have $\lim _{T \searrow T_{m}} B_{i}^{2}(T)=\left(2 p-\delta q_{0}\right)\left(\frac{\theta_{i}}{\gamma}-1\right) e^{\delta T_{m}}=p \frac{\theta_{i}-\gamma}{\gamma}$, and we also have $B_{i}^{2}\left(T_{x}\right)=$ $\left(2 p-\delta q_{0}\right)\left(\frac{2 \theta_{i}}{\gamma}-1\right) e^{\delta T_{m}}=p \frac{2 \theta_{i}-\gamma}{\gamma} \geq 0$ as $\theta_{i} \geq \frac{\gamma}{2}$ for the interior solution.

- In the case where $\theta_{i} \geq \gamma$, the marginal benefit of conceding is positive when $T$ tends towards $T_{m}$, and it is increasing in $T$.

- In the case where $\frac{\gamma}{2} \leq \theta_{i}<\gamma$, the marginal benefit of conceding of country $i$ is strictly negative when $T$ tends towards $T_{m}$ but positive at $T_{x}$, and so there exists a date $\widehat{T}_{i}=$ 
$T_{m}+\frac{1}{\delta}\left(\ln \gamma-\ln \theta_{i}\right)$ from which it becomes positive. The optimal date of concession is compulsorily greater than $\widehat{T}_{i}$.

We now characterize more precisely the optimal date of concession.

\subsubsection{The symmetric Nash equilibrium}

We consider, as in the first game, the symmetric Nash equilibrium in which the two countries concede following the same function $T(\theta)$.

Proposition 2 If the parameters of preference for the environment are uniformly distributed on $[\underline{\theta}, \bar{\theta}]$, the symmetric Nash equilibrium of the second game (the game in which one country cannot stabilize environmental quality alone) has the following characteristics:

(i) an interior solution exists for $\theta \in\left[\max \left\{\underline{\theta}, \frac{\gamma}{2}\right\}, \max \left\{\theta_{m}, \frac{\gamma}{2}\right\}[\right.$;

(ii) for $\theta \in\left[\min \left\{\underline{\theta}, \frac{\gamma}{2}\right\}, \min \left\{\theta_{m}, \frac{\gamma}{2}\right\}\left[\right.\right.$, the solution is the corner solution $T(\theta)=T_{x}$;

(iii) in the case of an interior solution, the boundary condition writes $T\left(\theta_{m}-\varepsilon\right)=T_{m}$ iff $\theta_{m} \geq \gamma$, and $T\left(\theta_{m}-\varepsilon\right)=\widehat{T}_{m}$ iff $\theta_{m}<\gamma ;$ the concession function is then decreasing from $T_{x}$ to $T_{m}$ or $\widehat{T}_{m}$, and the slope of the function for a parameter $\theta_{m}-\varepsilon$ is greater in absolute value than the slope of the concession function of the first game at $\theta_{m}$.

Proof. If $\underline{\theta}<\frac{\gamma}{2}$, we have shown above that the relevant solution is the corner solution for $\theta \in\left[\underline{\theta}, \max \left\{\theta_{m}, \frac{\gamma}{2}\right\}\left[\right.\right.$, and the interior one for $\theta \in\left[\frac{\gamma}{2}, \max \left\{\theta_{m}, \frac{\gamma}{2}\right\}[\right.$. This proves (i) and (ii).

If $\underline{\theta} \geq \frac{\gamma}{2}$, the relevant solution is the interior one for $\theta \in\left[\underline{\theta}, \theta_{m}[\right.$. Then a country with a parameter of preference equal to $\theta_{m}-\varepsilon$ (with $\varepsilon \rightarrow 0$ ) knows with certainty that it has the highest possible parameter of preference. We have shown above that besides, its incentive to concede at $T_{m}$ is positive if and only if $\theta_{m} \geq \gamma$, and strictly negative otherwise. Remark 1 then tells us that this country will decide to reduce its pollution at once $\left(T\left(\theta_{m}-\varepsilon\right)=T_{m}\right)$ if and only if $\theta_{m} \geq \gamma$, and to reduce it as soon as its benefit becomes positive $\left(T\left(\theta_{m}-\varepsilon\right)=\widehat{T}_{m}\right)$ if and only if $\theta_{m}<\gamma$. 
For $\underline{\theta} \geq \frac{\gamma}{2}$, in the case of the symmetric Nash equilibrium we show that

$$
T^{\prime}(\theta)=-\frac{f(\theta)}{F(\theta)} \frac{1}{B(T(\theta))}=-\frac{f(\theta)}{F(\theta)} \frac{e^{\delta T_{x}}-e^{\delta T(\theta)}}{\theta e^{\delta T(\theta)}-\gamma e^{\delta T_{m}}},
$$

with $T^{\prime}(\theta)<0$ by definition of an interior solution, which yields, when the preference parameters are uniformly distributed,

$$
T^{\prime}(\theta)=\frac{1}{\theta-\underline{\theta}} \frac{e^{\delta T_{x}}-e^{\delta T(\theta)}}{\gamma e^{\delta T_{m}}-\theta e^{\delta T(\theta)}} .
$$

This equation cannot be integrated with elementary functions. A numerical study, with the initial condition $\lim _{\theta / \theta_{m}} T(\theta)=T_{m}$ when $\theta_{m} \geq \gamma$ and $\lim _{\theta} \gamma_{m} T(\theta)=\widehat{T}_{m}$ otherwise, shows that the solution is a continuous and decreasing function $T(\theta)$, with $T(\underline{\theta})=T_{x}$.

When $\theta_{m}>\gamma$, one gets

$$
\lim _{\theta \nearrow \theta_{m}} T^{\prime}(\theta)=-\frac{1}{\left(\theta_{m}-\underline{\theta}\right)\left(\theta_{m}-\gamma\right)}
$$

whereas when concession occurs before $T_{m}$ one gets

$$
T^{\prime}\left(\theta_{m}\right)=-\frac{1}{\left(\theta_{m}-\underline{\theta}\right)\left(\theta_{m}+\delta-\gamma\right)}
$$

So the slope of the function of concession is then greater in absolute value before $\theta_{m}$ than after. This proves (iii).

\subsection{A comparison of intertemporal welfares}

We must now determine if, when no interior solution exists in the first game, country $i$ must concede at $T_{m}$ or play the second game.

Proposition 3 The expected intertemporal welfare obtained by playing the second game is always greater than the one obtained by choosing the corner solution of the first game. This ensures that in the absence of an interior solution to the first game, country $i$ will not choose to reduce its pollution at $T_{m}$ but will postpone further stabilization.

The proof of this proposition is tedious and therefore we relegate it to Appendix $\mathrm{C}^{12}$.

\footnotetext{
${ }^{12}$ See footnote 7 .
} 


\subsection{Summary of the results}

Our results are summed up in Figure 1, which shows the concession function on the whole range $[\underline{\theta}, \bar{\theta}]$, and therefore the solution of the stabilization game.

For $\theta \in\left[\theta_{m}, \bar{\theta}\right]$, the optimal stabilization date is the interior solution of the first game, in which the greenest country stabilizes alone; it is a decreasing function of $\theta$, from $T_{m}$ to 0 , and it is convex (Proposition 1).

So stabilization by a single country, the greenest, occurs only if this country is green enough, which means that it has a parameter of preference for the environment greater than $\theta_{m}$. The value of $\theta_{m}$ itself depends on the BAU emissions of pollution, the initial environmental quality and the impatience of the countries. The worst the initial conditions and the more impatient the countries, the greener the greenest country has to be. The higher $\theta$, the faster the stabilization of environmental quality.

For $\theta \in\left[\underline{\theta}, \theta_{m}[\right.$, the optimal stabilization date is the solution of the second game, in which both countries reduce their pollution (Proposition 3). Three cases can then occur (Proposition 2). They are depicted in Figure 1, where they are obtained by making the discount rate $\gamma$ vary, other things being equal.

In case (a), impatience is low $\left(\gamma<\theta_{m}\right.$ and $\left.\gamma / 2<\underline{\theta}\right)$, and the configuration of parameters such that in the second game the interior solution prevails. The optimal stabilization is a decreasing function of $\theta$, from $T_{x}$ to $T_{m}$. We could not obtain a formal result for the concavity of this function, but numerical simulations show us that it is concave most of the time.

In case (b), impatience is medium $\left(\gamma<\theta_{m}\right.$ but $\left.\gamma / 2>\underline{\theta}\right)$, and the configuration of parameters such that there exists an accumulation of concessions at $T_{x}$. If the greenest country has a parameter of preference for the environment between $\underline{\theta}$ and $\frac{\gamma}{2}$, both will rationally let the natural capital reach its minimum level.

Finally, in case (c), impatience is high $\left(\gamma>\theta_{m}\right.$ and $\left.\gamma / 2>\underline{\theta}\right)$, and the configuration of parameters such that there exists an accumulation of concessions at $T_{x}$ but also a jump in the concession function for $\theta_{m}$. Between $T_{m}$ and $\widehat{T}_{m}$, no country will rationally decide to concede, 
whatever its parameter of preference for the environment.

So if the greenest country is not green enough, both countries wait until it is impossible for one of them to stabilize alone. Then the occurence of the stabilization depends on the comparison of the smallest preference for the environment $\underline{\theta}$ and the half of the discount rate. If impatience is so low that $\frac{\gamma}{2} \leq \underline{\theta}$, the greenest country reduces its pollution to zero and the other one does the rest of the job of stabilization. When impatience is high $\left(\frac{\gamma}{2}>\underline{\theta}\right)$, the same thing occurs if the greenest country is green enough $\left(\theta>\frac{\gamma}{2}\right)$, but a complete depletion of environmental quality is optimal if it is not the case.

\section{Conclusion}

Due to the absence of a supra-national authority able to monitor the emergence and implementation of a coordinated pollution abatement program, the world is likely to be trapped in lasting excessive pollution, due to non-cooperative national policies. We have shown, in the war of attrition framework, that the excess of pollution will be all the more persistent since the greenest of the two countries is less green, the initial conditions are adverse (high business-as-usual pollution and low initial environmental quality), the countries are optimistic concerning the possible range of the preference parameters, and impatience corrected for natural regeneration is high. We have also shown that it can be rational for the greenest country to wait so long that it cannot stabilize alone. Furthermore, it is also possible that stabilization never occurs.

Natural possible extensions are the following. First, the representation of the economy, the environment, and their interactions could be less oversimplified. For instance, empirical studies show that the regeneration rate is not a constant but depends on the level of environmental quality itself. If natural regeneration slows down as environmental quality diminishes, the threshold constituted by impatience corrected for natural regeneration increases, making stabilization more likely. Secondly, we could introduce more asymmetry between the players, which are here identical except for their preference for the environment. For instance, they could have different social discount rates (one country could be more impatient than the other), or different 
business-as-usual levels of polluting activities. Thirdly, it would obviously be very interesting to extend this framework to the case of more than two countries, in the spirit of generalized war of attrition models ( $c f$. Bulow and Klemperer, 1999). The global warming problem for example could be better studied in a framework considering three different groups of countries, the United States, the signatories of the Kyoto Protocol, and the developing countries. 


\section{References}

Alesina, A. and A. Drazen, 1991, Why are stabilizations delayed?, American Economic Review 81(5), 1170-1188.

Bac, M., 1996, Incomplete information and incentives to free ride on international environmental resources, Journal of Environmental Economics and Management 30, 301-315.

Barrett, S., 1994, Self-enforcing international environmental agreements, Oxford Economic Papers $46,878-894$.

Barrett, S., 1998, Political economy of the Kyoto Protocol, Oxford Review of Economic Policy 14(4), 20-39.

Bliss, C. and B. Nalebuff, 1984, Dragon-slaying and ballroom dancing: the private supply of a public good, Journal of Public Economics 25(1-2), 1-12.

Bulow, J. and P. Klemperer, 1999, The generalized war of attrition, American Economic Review 89(1), 175-189.

Carraro, C. and D. Siniscalco, 1993, Strategies for the international protection of environment, Journal of Public Economics 52(3), 309-328.

Carré, M., 2000, Debt stabilization with a deadline, European Economic Review 44, 71-90.

Casella, A. and Eichengreen, 1996, Can foreign aid accelerate stabilization?, The Economic Journal 106, 605-619.

Chander, P. and H. Tulkens, 1992, Theoretical foundations of cost sharing in transfrontier pollution problems, European Economic Review 36, 388-399.

Chander, P. and H. Tulkens, 1995, A core-theoretic solution for the design of cooperative agreements on transfrontier pollution, International Tax and Public Finance 2, 273-294.

Dockner, E.J. and N. van Long, 1993, International pollution control, cooperative versus noncooperative strategies, Journal of Environmental Economics and Management 25, 13-29.

Hoël, M., 1992, International environmental conventions: the case of uniform reductions of emissions, Environmental and Resource Economics 2, 141-159. 

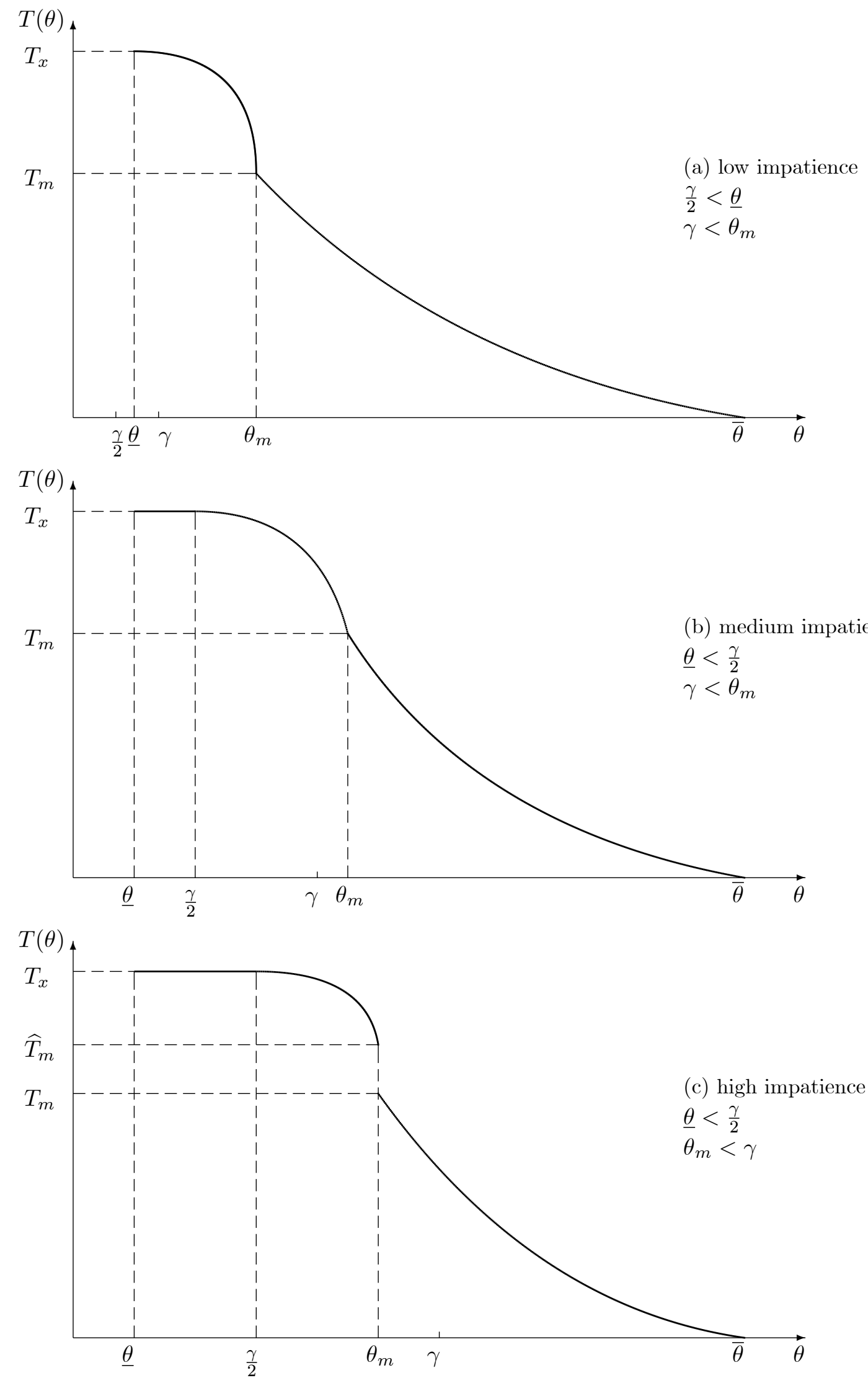

Figure 1: The optimal stabilization date 